Int. J. Electrochem. Sci., 11 (2016) $9614-9624$

\title{
Deposition of Au Nanoparticles onto Poly(3,4-ethylenedioxy - thiophene) Functionalized Multi-Walled Carbon Nanotubes for Label-Free Immunosensing
}

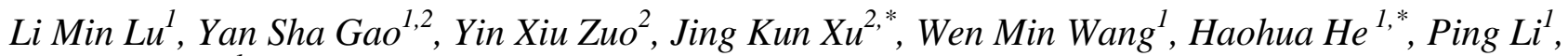 \\ Yong Fang $\mathrm{Yu}^{\mathrm{l}}$ \\ ${ }^{1}$ College of Science, Jiangxi Agricultural University, Nanchang 330045, PR China \\ 2 Jiangxi Key Laboratory of Organic Chemistry, Jiangxi Science and Technology Normal University, \\ Nanchang 330013, PR China \\ *E-mail: xujingkun@tsinghua.org.cn, hhhua64@163.com
}

doi: $10.20964 / 2016.11 .11$

Received: 15 July 2016 / Accepted: 29 August 2016 / Published: 10 October 2016

\begin{abstract}
A high performance electrochemical immunosensor was constructed for sensitive label-free detection for $\alpha$-fetoprotein ( $\alpha$-FP) based on Au nanoparticles/poly(3,4-ethylenedioxythiophene) modified multiwalled carbon nanotubes (AuNPs/PEDOT-MWCNTs), which acted as signal amplification matrix. PEDOT functionalized MWCNTs were prepared via interfacial polymerization method. PEDOT was uniformly distributed on MWCNTs, which can provide more binding sites for high mass loading of metal nanoparticles. Subsequently, AuNPs were attached on MWCNTs by introducing conductive PEDOT to bridge the AuNPs and MWCNTs walls. The AuNPs/PEDOT-MWCNTs nanocomposites displayed good electron transfer ability, which enabled good analytical properties for $\alpha$-FP detection.
\end{abstract}

Keywords: Electrochemical method; Tumor marker; Poly(3,4-ethylenedioxythiophene) nanocomposites; High sensitivity

\section{$\underline{\text { FULL TEXT }}$}

(C) 2016 The Authors. Published by ESG (www.electrochemsci.org). This article is an open access article distributed under the terms and conditions of the Creative Commons Attribution license (http://creativecommons.org/licenses/by/4.0/). 